\title{
THE SOLUTION OF CERTAIN TRIPLE INTEGRAL EQUATIONS INVOLVING INVERSE MELLIN TRANSFORMS $\dagger$
}

\author{
by J. TWEED
}

(Received 9 June, 1971)

1. Introduction. In this paper, we shall be concerned with the solution of triple integral equations of the type

$$
\left.\begin{array}{cl}
\mathscr{M}^{-1}\left[(1+s)^{-1} A(s) ; r\right]=0 & (0<r<a), \\
\mathscr{M}^{-1}[\cot (\pi s / n) A(s) ; r]=f(r) & (a<r<b), \\
\mathscr{M}^{-1}\left[(1+s)^{-1} A(s) ; r\right]=0 & (b<r<\infty),
\end{array}\right\}
$$

where $\mathscr{H}^{-1}$ is the inverse Mellin transform, $n$ is a positive integer, and $-1<\operatorname{Re} s<0$. The use of these equations will be illustrated by their application to two well-known problems in the mathematical theory of elasticity and further applications will be reported later.

2. We begin by considering the equations

$$
\left.\begin{array}{rlrl}
\mathscr{M}^{-1}\left[(1+s)^{-1} A(s) ; r\right] & =0 & & (0<r<a), \\
\mathscr{M}^{-1}[\cot (\pi s) A(s) ; r] & =f(r) & & (a<r<b), \\
\mathscr{M}^{-1}\left[(1+s)^{-1} A(s) ; r\right] & =0 & & (b<r<\infty),
\end{array}\right\}
$$

where $-1<\operatorname{Re} s<0$. The method of solution is similar to that used by Lowengrub and Srivastava [1] for the solution of triple integral equations with trigonometric kernels and involves the assumption of a solution of the form

$$
A(s)=\int_{a}^{b} p(t) t^{s+1} d t
$$

where $p(t)$ is an unknown function to be determined later. It is clear that, with this choice of $A(s)$,

$$
\mathscr{A}^{-1}\left[(1+s)^{-1} A(s) ; r\right]=\int_{a}^{b} t p(t) \mathscr{M}^{-1}\left[(1+s)^{-1} ; r / t\right] d t
$$

and therefore, since

$$
\mathscr{M}^{-1}\left[(1+s)^{-1} ; r\right]=r H(1-r) \quad(\operatorname{Re} s>-1),
$$

$\dagger$ This paper was prepared as a part of the work of the Applied Mathematics Research Group at North Carolina State University through the Grant AF-AFOSR-69-1779 and is under joint sponsorship of AFOSR, $A R O$ and ONR through the Joint Services Advisory Group.

E 
we see that, if $-1<\operatorname{Re} s<0$, then

$$
\mathscr{A}^{-1}\left[(1+s)^{-1} A(s) ; r\right]=\left\{\begin{array}{cc}
r \int_{a}^{b} p(t) d t & (0<r<a), \\
r \int_{r}^{b} p(t) d t & (a<r<b), \\
0 & (b<r<\infty) .
\end{array}\right\}
$$

It follows that the first and third of the equations (2.1) will be satisfied automatically by (2.2) provided that

$$
\int_{a}^{b} p(t) d t=0
$$

Similarly, on making use of the result

$$
\mathscr{H}^{-1}[\pi \cot (\pi s) ; r]=r(1-r)^{-1} \quad(-1<\operatorname{Re} s<0),
$$

we see that

$$
\mathscr{M}^{-1}[\cot (\pi s) A(s) ; r]=\frac{r}{\pi} \int_{a}^{b} \frac{t p(t) d t}{t-r}
$$

and therefore the second of the equations (2.1) will be satisfied if $p(t)$ is chosen to be the solution of the integral equation

$$
\frac{1}{\pi} \int_{a}^{b} \frac{t p(t) d t}{t-r}=\frac{f(r)}{r} \quad(a<r<b) .
$$

The solution of equation (2.6) is well known (see Tricomi [3]) and may be written in either of the two alternative forms

$$
t p(t)=\frac{-1}{\pi}\left(\frac{t-a}{b-t}\right)^{\frac{1}{2}} \int_{a}^{b}\left(\frac{b-y}{y-a}\right)^{\frac{1}{2}} \frac{f(y)}{y-t} \frac{d y}{y}+\frac{C_{1}}{[(t-a)(b-t)]^{\frac{1}{2}}}
$$

or

$$
t p(t)=\frac{-1}{\pi}\left(\frac{b-t}{t-a}\right)^{\frac{1}{2}} \int_{a}^{b}\left(\frac{y-a}{b-y}\right)^{\frac{t}{t}} \frac{f(y)}{y-t} \frac{d y}{y}+\frac{C_{2}}{[(t-a)(b-t)]^{\frac{1}{2}}},
$$

where $C_{1}$ and $C_{2}$ are constants which are determined by the condition (2.4). On applying this condition it is seen, after a little manipulation, that

and

$$
C_{1}=-\frac{a}{\pi} \int_{a}^{b}\left(\frac{b-y}{y-a}\right)^{\frac{1}{2}} \frac{f(y)}{y^{2}} d y
$$

$$
C_{2}=\frac{b}{\pi} \int_{a}^{b}\left(\frac{y-a}{b-y}\right)^{\frac{1}{2}} \frac{f(y)}{y^{2}} d y,
$$

thus completing the solution of the problem. 
In those physical problems that can be reduced to the solution of equations such as (2:1) we are usually interested in finding not only the solution of the equations but the values taken by their left-hand sides in the intervals on which they are not prescribed. For completeness we shall now do this. On substituting from (2.7) and (2.8) into (2.5) and changing the order of integration it is easily seen that we may write

$$
\mathscr{H}^{-1}[\cot (\pi s) A(s) ; r]=\left\{\begin{array}{cc}
-\frac{r}{\pi}\left(\frac{b-r}{a-r}\right)^{\frac{1}{2}} \int_{a}^{b}\left(\frac{y-a}{b-y}\right)^{\frac{1}{2}} \frac{f(y)}{y-r} \frac{d y}{y}+\frac{r C_{2}}{[(a-r)(b-r)]^{\frac{1}{2}}} & (r<a), \\
\frac{r}{\pi}\left(\frac{r-a}{r-b}\right)^{\frac{1}{2}} \int_{a}^{b}\left(\frac{b-y}{y-a}\right)^{\frac{1}{2}} \frac{f(y)}{y-r} \frac{d y}{y}-\frac{r C_{1}}{[(r-a)(r-b)]^{\frac{1}{2}}} & (r>b),
\end{array}\right.
$$

and on substituting the values of $C_{1}$ and $C_{2}$ given by (2.9) and (2.10) into these expressions we find that

$$
\mathscr{H}^{-1}[\cot (\pi s) A(s) ; r]=\left\{\begin{array}{ll}
\frac{r^{2}}{\pi[(a-r)(b-r)]^{\frac{1}{t}}} \int_{a}^{b} \frac{[(y-a)(b-y)]^{\frac{1}{2}}}{r-y} \frac{f(y)}{y^{2}} d y & (r<a), \\
\frac{r^{2}}{\pi[(r-a)(r-b)]^{\frac{1}{2}}} \int_{a}^{b} \frac{[(y-a)(b-y)]^{\frac{1}{2}}}{r-y} \frac{f(y)}{y^{2}} d y & (r>b) .
\end{array}\right\}
$$

Similarly, on substituting from (2.7) and (2.9) into (2.3) and performing some manipulation, it can be shown that, for $a<r<b$,

$$
\mathscr{H}^{-1}\left[(1+s)^{-1} A(s) ; r\right]=\frac{r}{\pi} \int_{r}^{b} \frac{d t}{[(t-a)(b-t)]^{\frac{1}{2}}} \int_{a}^{b} \frac{[(b-y)(y-a)]^{\frac{1}{2}}}{t-y} \frac{f(y)}{y^{2}} d y .
$$

3. We shall now turn our attention to the equations

$$
\left.\begin{array}{ll}
\mathscr{M}^{-1}\left[(1+s)^{-1} A(s) ; r\right]=0 & (0<r<a), \\
\mathscr{M}^{-1}[\cot (\pi s / n) A(s) ; r]=f(r) & (a<r<b), \\
\mathscr{M}^{-1}\left[(1+s)^{-1} A(s) ; r\right]=0 & (b<r<\infty)
\end{array}\right\}
$$

where $-\ddot{1}<\operatorname{Re} s<0$, and $n \geqq 2$. In this case we shall assume a solution of the form

$$
A(s)=\int_{a}^{b} p\left(t^{n}\right) t^{s+n-1} d t
$$

As before, we see that

$$
\mathscr{H}^{-1}\left[(1+s)^{-1} A(s) ; r\right]=\left\{\begin{array}{cc}
r \int_{a}^{b} t^{n-2} p\left(t^{n}\right) d t & (0<r<a), \\
r \int_{r}^{b} t^{n-2} p\left(t^{n}\right) d t & (a<r<b), \\
0 & (b<r<\infty)
\end{array}\right\}
$$


so that the first and third of the equations (3.1) will be satisfied if

$$
\int_{a}^{b} t^{n-2} p\left(t^{n}\right) d t=0
$$

If we now substitute the expression (3.2) into the left-hand side of the second equation we find that

$$
\mathscr{M}^{-1}[\cot (\pi s / n) A(s) ; r]=\frac{1}{\pi} \int_{a}^{b} t^{n-1} p\left(t^{n}\right) \mathscr{M}^{-1}[\pi \cot (\pi s / n) ; s \rightarrow r / t] d t
$$

and therefore, on making use of the result

$$
\mathscr{M}^{-1}[\pi \cot (\pi s / n) ; s \rightarrow r]=n r^{n}\left(1-r^{n}\right)^{-1} \quad(-1<\operatorname{Re} s<0) ;
$$

we see that, if $-1<\operatorname{Re} s<0$,

$$
\mathscr{M}^{-1}[\cot (\pi s / n) A(s) ; r]=\frac{n r^{n}}{\pi} \int_{a}^{b} \frac{t^{n-1}}{t^{n}-r^{n}} p\left(t^{n}\right) d t .
$$

It follows that the second equation will be satisfied if $p\left(t^{n}\right)$ is the solution of the integral equation

$$
\frac{1}{\pi} \int_{a}^{b} \frac{n t^{n-1}}{t^{n}-r^{n}} p\left(t^{n}\right) d t=\frac{f(r)}{r^{n}} \quad(a<r<b) .
$$

By making a simple change of variable, this equation can be transformed into one similar to (2.6) and therefore it is easily seen that its solution may be written in either of the forms

$$
p\left(t^{n}\right)=-\frac{n}{\pi}\left(\frac{t^{n}-a^{n}}{b^{n}-t^{n}}\right)^{\frac{1}{2}} \int_{a}^{b}\left(\frac{b^{n}-y^{n}}{y^{n}-a^{n}}\right)^{\frac{1}{2}} \frac{f(y)}{y^{n}-t^{n}} \frac{d y}{y}+\frac{C_{1}}{\left[\left(t^{n}-a^{n}\right)\left(b^{n}-t^{n}\right)\right]^{\frac{1}{2}}}
$$

or

$$
p\left(t^{n}\right)=-\frac{n}{\pi}\left(\frac{b^{n}-t^{n}}{t^{n}-a^{n}}\right)^{\frac{1}{2}} \int_{a}^{b}\left(\frac{y^{n}-a^{n}}{b^{n}-y^{n}}\right)^{\frac{1}{2}} \frac{f(y)}{y^{n}-t^{n}} \frac{d y}{y}+\frac{C_{2}}{\left[\left(t^{n}-a^{n}\right)\left(b^{n}-t^{n}\right)\right]^{\frac{1}{2}}},
$$

where $C_{1}$ and $C_{2}$ are constants to be determined from the condition (3.4). The application of this condition yields the results

$$
C_{1}=\frac{n}{\pi I_{n}} \int_{a}^{b}\left(\frac{b^{n}-y^{n}}{y^{n}-a^{n}}\right)^{\frac{t}{f}} \frac{f(y)}{y} d y \int_{a}^{b}\left(\frac{t^{n}-a^{n}}{b^{n}-t^{n}}\right)^{t} \frac{t^{n-2}}{y^{n}-t^{n}} d t
$$

and

$$
C_{2}=\frac{n}{\pi I_{n}} \int_{a}^{b}\left(\frac{b^{n}-t^{n}}{t^{n}-a^{n}}\right)^{\frac{1}{2}} t^{n-2} d t \int_{a}^{b}\left(\frac{y^{n}-a^{n}}{b^{n}-y^{n}}\right)^{t} \frac{f(y)}{y^{n}-t^{n}} \frac{d y}{y},
$$

where $I_{n}$ is the integral

$$
\int_{a}^{b} t^{n-2}\left[\left(t^{n}-a^{n}\right)\left(b^{n}-t^{n}\right)\right]^{-\frac{1}{2}} d t
$$


In this case, it is easily shown that a convenient way of writing the left-hand side of the second equation is

$$
\begin{aligned}
\mathscr{H}^{-1}[\cot (\pi s / n) A(s) ; r] \\
=\left\{\begin{aligned}
\frac{n r^{n}}{\pi}\left(\frac{r^{n}-a^{n}}{r^{n}-b^{n}}\right)^{\frac{1}{2}} \int_{a}^{b}\left(\frac{b^{n}-y^{n}}{y^{n}-a^{n}}\right)^{\frac{1}{2}} \frac{f(y)}{y^{n}-r^{n}} \frac{d y}{y}-\frac{C_{1} r^{n}}{\left[\left(r^{n}-a^{n}\right)\left(r^{n}-b^{n}\right)\right]^{\frac{1}{t}}} & (r>b), \\
\frac{-n r^{n}}{\pi}\left(\frac{b^{n}-r^{n}}{a^{n}-r^{n}}\right)^{\frac{1}{2}} \int_{a}^{b}\left(\frac{y^{n}-a^{n}}{b^{n}-y^{n}}\right)^{\frac{1}{2}} \frac{f(y)}{y^{n}-r^{n}} \frac{d y}{y}+\frac{C_{2} r^{n}}{\left[\left(a^{n}-r^{n}\right)\left(b^{n}-r^{n}\right)\right]^{\frac{1}{t}}} & (r<a) .
\end{aligned}\right\}
\end{aligned}
$$

There appears however, to be no such simple formula for the left-hand side of the first and third equations.

4. In this section, we show how the results of the preceding sections may be used to find the solutions of two well-known problems in the two-dimensional theory of elasticity under the assumption of plane strain.

Problem 1. The first problem we consider is that of finding the stress intensity factors and crack shape of a Griffith crack which is defined, in plane polar co-ordinates, by the relations $\theta=0, a<r<b$ and whose surfaces are loaded symmetrically. Due to symmetry, this problem reduces to that of finding the solution of the equations of elasticity for the half plane $0 \leqq r<\infty, 0 \leqq \theta \leqq \pi$ which satisfies the conditions

(1) $\sigma_{r \theta}(r, \pi)=v(r, \pi)=0 \quad(0 \leqq r<\infty)$,

(2) $\sigma_{r \theta}(r, 0)=0 \quad(0 \leqq r<\infty)$,

(3) $\sigma_{\theta}(r, 0)=-f(r) \quad(a<r<b)$,

(4) $v(r, 0)=0 \cdot(0 \leqq r<a) \cup(b<r<\infty)$,

(5) All the stresses and displacements are bounded at the origin and tend to zero as $r$ tends to infinity.

But utilizing the properties of the Mellin transform, it can be shown that the solution of the equations of elasticity in plane polar coordinates that satisfies condition (5) is such that

and

$$
\begin{aligned}
& \frac{r^{2}}{2 \mu} \sigma_{\theta}(r, \theta)=\mathscr{M}^{-1}[s(s+1) \bar{\chi}(s, \theta) ; r], \\
& \frac{r^{2}}{2 \mu} \sigma_{r \theta}(r, \theta)=\mathscr{M}^{-1}\left[(s+1) \frac{d \bar{\chi}(s, \theta)}{d \theta} ; r\right]
\end{aligned}
$$

$r v(r, \theta)=\mathscr{M}^{-1}\left[\frac{-1}{(s+1)(s+2)}\left\{(1-\eta) \frac{d^{3} \bar{\chi}(s, \theta)}{d \theta^{3}}+\left[(1-\eta) s^{2}+(s+1)(s+2)\right] \frac{d \bar{\chi}(r, \theta)}{d \theta}\right\} ; r\right]$,

where $-1<\operatorname{Re} s<0$, and $\bar{\chi}(s, \theta)$, which is the Mellin transform of the Airy stress function, is given by

$$
\bar{\chi}(s, \theta)=C_{1} \sin s \theta+C_{2} \cos s \theta+C_{3} \sin (s+2) \theta+C_{4} \cos (s+2) \theta
$$

(see Tranter [2]). 
If we now put

$$
\begin{array}{ll}
C_{1}=\frac{(s+2) A}{4 s(s+1)}, & C_{2}=\frac{(s+2) A}{4 s(s+1)} \cot (\pi s), \\
C_{3}=-\frac{A}{4(s+1)}, & C_{4}=-\frac{A}{4(s+1)} \cot (s+2) \pi,
\end{array}
$$

it is easily shown that the conditions (1) and (2) are satisfied automatically and that

$$
\sigma_{\theta}(r, 0)=\frac{\mu}{r^{2}} \mathscr{M}^{-1}[A(s) \cot (\pi s) ; r]
$$

and

$$
v(r, 0)=-\frac{1-\eta}{r} \mathscr{M}^{-1}\left[(1+s)^{-1} A(s) ; r\right] .
$$

It now follows that the remaining boundary conditions will be satisfied if $A(s)$ is the solution of the triple integral equations

$$
\left.\begin{array}{rlrl}
\mathscr{M}^{-1}\left[(1+s)^{-1} A(s) ; r\right] & =0 & & (0<r<a), \\
\mathscr{M}^{-1}[\cot (\pi s) A(s) ; r] & =-\mu^{-1} r^{2} f(r) & & (a<r<b), \\
\mathscr{M}^{-1}\left[(1+s)^{-1} A(s) ; r\right] & =0 & & (b<r<\infty)
\end{array}\right\}
$$

where $-1<\operatorname{Re} s<0$.

These equations are of the type studied in $\$ 2$ and therefore we can conclude that

$$
\sigma_{\theta}(r, 0)=\left\{\begin{array}{ll}
\frac{1}{\pi[(a-r)(b-r)]^{\frac{1}{2}}} \int_{a}^{b} \frac{[(y-a)(b-y)]^{\frac{1}{3}}}{y-r} f(y) d y & (r<a), \\
\frac{1}{\pi[(r-a)(r-b)]^{\frac{1}{2}}} \int_{a}^{b} \frac{[(y-a)(b-y)]^{ \pm}}{r-y} f(y) d y & (r>b),
\end{array}\right\}
$$

and that

$$
v(r, 0)=-\frac{(1-\eta)}{\pi \mu} \int_{r}^{b} \frac{d t}{[(t-a)(b-t)]^{\frac{1}{2}}} \int_{a}^{b} \frac{[(b-y)(y-a)]^{\frac{1}{2}}}{y-t} f(y) d y,
$$

for $a \leqq r \leqq b$.

Of interest to workers in fracture mechanics are the stress intensity factors $K_{a}$ and $K_{b}$ which are defined by the relations

$$
K_{a}=\lim _{r \rightarrow a-}(a-r)^{\frac{1}{t}} \sigma_{\theta}(r, 0)
$$

and

$$
K_{b}=\lim _{r \rightarrow b+}(r-b)^{\frac{1}{2}} \sigma_{\theta}(r, 0)
$$


From the result (4.7) above it is clear that in this case

$$
K_{a}=\frac{1}{\pi(b-a)^{\frac{1}{2}}} \int_{a}^{b}\left(\frac{b-y}{y-a}\right)^{\frac{1}{t}} f(y) d y
$$

and

$$
K_{b}=\frac{1}{\pi(b-a)^{\frac{1}{2}}} \int_{a}^{b}\left(\frac{y-a}{b-y}\right)^{\frac{1}{2}} f(y) d y .
$$

Problem 2. The second problem we deal with is that of finding the stress intensity factors of a pair of Griffith cracks whose surfaces are loaded symmetrically and are defined by the relations $\theta=0, a<r<b ; \theta=\pi, a<r<b$. In this case, the symmetry in the problem leads us to seek a solution of the equations of elasticity for the quarter plane $0 \leqq r<\infty, 0 \leqq \theta \leqq \pi / 2$ which satisfies the conditions

(1) $\sigma_{r \theta}(r, \pi / 2)=v(r, \pi / 2)=0 \quad(0 \leqq r<\infty)$,

(2) $\sigma_{r \theta}(r, 0)=0 \quad(0 \leqq r<\infty)$,

(3) $\sigma_{\theta}(r, 0)=-f(r) \quad(a<r<b)$,

(4) $\quad v(r, 0)=0 \quad(0<r<a) \cup(b<r<\infty)$,

(5) All the stresses and displacements are bounded at the origin and tend to zero as $r$ tends to infinity.

As in Problem 1 above it is easily shown that the solution of the equations of elasticity satisfying conditions (1), (2) and (5) is such that

$$
\begin{aligned}
& \frac{r^{2}}{\mu} \sigma_{\theta}(r, 0)=\mathscr{A}^{-1}[\cot (\pi s / 2) A(s) ; r], \\
& \frac{r}{1-\eta} v(r, 0)=-\mathscr{A}^{-1}\left[(1+s)^{-1} A(s) ; r\right],
\end{aligned}
$$

where $-1<\operatorname{Re} s<0$.

It follows that conditions (3) and (4) will be satisfied if $A(s)$ is the solution of the triple integral equations

$$
\left.\begin{array}{ll}
\mathscr{M}^{-1}\left[(1+s)^{-1} A(s) ; r\right]=0 & (0<r<a), \\
\mathscr{M}^{-1}[\cot (\pi s / 2) A(s) ; r]=-\mu^{-1} r^{2} f(r) & (a<r<b), \\
\mathscr{H}^{-1}\left[(1+s)^{-1} A(s) ; r\right]=0 & (b<r<\infty),
\end{array}\right\}
$$

where $-1<\operatorname{Re} s<0$. 
But these equations are of the type studied in $\S 3$ and therefore, by (3.9), (3.10), (3.11) and (4.13) we see that

$$
\sigma_{\theta}(r, 0)=\left\{\begin{array}{cc}
\frac{2}{\pi}\left(\frac{r^{2}-a^{2}}{r^{2}-b^{2}}\right)^{\frac{1}{2}} \int_{a}^{b}\left(\frac{b^{2}-y^{2}}{y^{2}-a^{2}}\right)^{\frac{1}{2}} \frac{y f(y)}{r^{2}-y^{2}} d y-\frac{\mu C_{1}}{\left[\left(r^{2}-a^{2}\right)\left(r^{2}-b^{2}\right)\right]^{\frac{1}{2}}} & (r>b), \\
\frac{-2}{\pi}\left(\frac{b^{2}-r^{2}}{a^{2}-r^{2}}\right)^{\frac{1}{2}} \int_{a}^{b}\left(\frac{y^{2}-a^{2}}{b^{2}-y^{2}}\right)^{\frac{1}{2}} \frac{y f(y)}{r^{2}-y^{2}} d y+\frac{\mu C_{2}}{\left[\left(a^{2}-r^{2}\right)\left(b^{2}-r^{2}\right)\right]^{\frac{1}{2}}} & (r<a),
\end{array}\right\}
$$

where

$$
\begin{aligned}
& C_{1}=\frac{2}{\pi \mu I_{2}} \int_{a}^{b}\left(\frac{b^{2}-y^{2}}{y^{2}-a^{2}}\right)^{\frac{t}{2}} y f(y) d y \int_{a}^{b}\left(\frac{t^{2}-a^{2}}{b^{2}-t^{2}}\right)^{\frac{1}{2}} \frac{d t}{t^{2}-y^{2}}, \\
& C_{2}=\frac{2}{\pi \mu I_{2}} \int_{a}^{b}\left(\frac{b^{2}-t^{2}}{t^{2}-a^{2}}\right)^{\frac{1}{2}} d t \int_{a}^{b}\left(\frac{y^{2}-a^{2}}{b^{2}-y^{2}}\right)^{\frac{1}{2}} \frac{y f(y)}{t^{2}-y^{2}} d y
\end{aligned}
$$

and

$$
I_{2}=\int_{a}^{b} \frac{d t}{\left[\left(t^{2}-a^{2}\right)\left(b^{2}-t^{2}\right)\right]^{\frac{1}{2}}}=F / b
$$

where $F$ is the complete elliptic integral $F\left[\pi / 2, b^{-1}\left(b^{2}-a^{2}\right)^{\frac{1}{2}}\right]$. It now follows easily that in this case

$$
K_{a}=\frac{2}{\pi}\left(\frac{b^{2}-a^{2}}{2 a}\right)^{\frac{1}{2}} \int_{a}^{b} \frac{y f(y) d y}{\left[\left(b^{2}-y^{2}\right)\left(y^{2}-a^{2}\right)\right]^{\frac{1}{2}}}+\frac{\mu C_{2}}{\left[2 a\left(b^{2}-a^{2}\right)\right]^{\frac{1}{2}}}
$$

and

$$
K_{b}=\frac{2}{\pi}\left(\frac{b^{2}-a^{2}}{2 b}\right)^{\frac{1}{2}} \int_{a}^{b} \frac{y f(y) d y}{\left[\left(b^{2}-y^{2}\right)\left(y^{2}-a^{2}\right)\right]^{\frac{1}{2}}}-\frac{\mu C_{1}}{\left[2 b\left(b^{2}-a^{2}\right)\right]^{\frac{1}{2}}} .
$$

\section{REFERENCES}

1. M. Lowengrub and K. N. Srivastava, On two coplanar cracks in an infinite elastic medium, Int. J. Eng. Sci. 6 (1968), 359-367.

2. C. J. Tranter, Integral transforms in mathematical physics (London, 1951).

3. F. A. Tricomi, On the finite Hilbert transformation, Quart. J. Mech. Appl. Math. 2 (1951), 199-211.

\section{UNIVERSITY OF GLASGOW}

GLASGOW, G12 8QQ 